\title{
Measurements of visible bremsstrahlung and automatic Bayesian inference of the effective plasma charge $Z_{\text {eff }}$ at W7-X
}

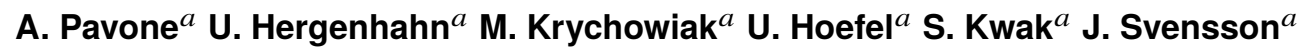 \\ P. Kornejew ${ }^{a}$ V. Winters ${ }^{b}$ R. Koenig ${ }^{a}$ M. Hirsch ${ }^{a}$ K.-J. Brunner ${ }^{a}$ E. Pasch $^{a}$ J. Knauer ${ }^{a}$

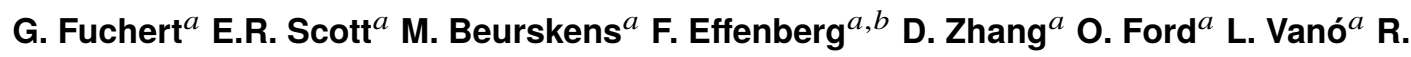 \\ C. Wolf ${ }^{a}$ W7-X Team ${ }^{a}$ \\ ${ }^{a}$ Max-Planck-Institut für Plasmaphysik, Teilinstitut Greifswald, D-17491 Greifswald, DE \\ ${ }^{b}$ University of Wisconsin-Madison, Madison, WI 53706-1609, USA \\ E-mail: andrea.pavone@ipp.mpg.de
}

Abstract: The effective charge $Z_{\text {eff }}$ indicates the overall impurity contamination of a plasma. $Z_{\text {eff }}$ can be derived experimentally from the intensity of the plasma bremsstrahlung emission. We describe here the diagnostic set-ups and the Bayesian modeling allowing the inference of $Z_{\text {eff }}$ at W7-X. First results from the operational campaigns in 2017 and 2018 are shown. Measurements of the visible plasma radiation along a single line-of-sight traversing the core plasma has been carried out using a compact USB-spectrometer with a time resolution of $100 \mathrm{~ms}$. A spectral region $(627-641 \mathrm{~nm})$ that is free from line emission is selected for the analysis of the bremsstrahlung emission, which also depends on electron temperature and density profiles. Electron temperature profiles are derived from either the electron cyclotron emission or the Thomson scattering diagnostic. Electron density profiles, however, have their shape information derived from Thomson scattering measurements and absolute values from single line-of-sight interferometry measurements. The Minerva framework is used to infer the profiles with Gaussian processes and develop a Bayesian model of the bremsstrahlung emission to infer line averaged $Z_{\mathrm{eff}}$. The sensitivity of the diagnostic enables $Z_{\text {eff }}$ measurements down to the lowest core electron densities observed in the last campaign of $0.75 \times 10^{19} \mathrm{~m}^{-3}$ with a statistical relative error of $\approx 50 \%\left(Z_{\mathrm{eff}}=3.2,100 \mathrm{~ms}\right.$ integration time). The analysis is automated to routinely compute $Z_{\mathrm{eff}}$ after every plasma discharges.

KeYwords: Zeff, bremsstrahlung, Bayesian inference, neural network, Minerva 


\section{Contents}

1 Introduction 1

2 The single line-of-sight USB-spectrometer diagnostic 1

3 Bayesian modeling and inference $\quad 2$

4 Results 4

5 Conclusions and future works $\quad 4$

\section{Introduction}

In magnetically confined fusion plasmas, the study of impurity behavior is important for the assessment of plasma performance and the investigation of impurity transport [1]. The effective charge $Z_{\text {eff }}=\sum_{i} n_{i} Z_{i}^{2} / \sum_{i} n_{i} Z_{i}$ is related to the concentration of impurities and indicates the overall contamination of the plasma with mainly low-Z impurities, e.g. Carbon. It is usually derived experimentally from the plasma ion-electron bremsstrahlung emission in the visible, IR or X-Ray spectral region [2-4], using an independent measurement of the electron density $n_{\mathrm{e}}$ and temperature $T_{\mathrm{e}}$. In this work, we illustrate the diagnostic set-ups and the Bayesian modeling that allowed the inference of $Z_{\text {eff }}$ at W7-X and we will show results from the OP1.2 experimental campaign, obtained from measurements performed with a compact USB-spectrometer. Also, we will describe the diagnostic set-up of other diagnostic systems which were routinely observing bremsstrahlung emission as well.

\section{The single line-of-sight USB-spectrometer diagnostic}

A compact USB-spectrometer (Red Tide USB650, Ocean Optics) collects light along a single line-of-sight that goes through the plasma core of W7-X, as shown in figure 1a. The system collects light in the visible and near infrared wavelength region, approximately from 350 to $1000 \mathrm{~nm}$, as shown in figure $1 \mathrm{~b}$, with a time resolution of $100 \mathrm{~ms}$. Due to the low light level of the calibration source only the spectral range above $450 \mathrm{~nm}$ can be used for the analysis. The figure also shows the bremsstrahlung emission predicted with $Z_{\mathrm{eff}} \approx 1.5$. Details about the predictive forward model are given in the following sections. In order to infer $Z_{\text {eff }}$ from the measured spectrum, we have selected and used a fixed wavelength window that is free of line radiation, marked with two red vertical lines in the figure, in the range of $\approx 627-641 \mathrm{~nm}$. The system was absolutely calibrated by measuring the diagnostic response to an Ulbricht sphere of known emissivity. The calibration has been carried out prior (pre), during (mid) and after (post) both the experimental campaigns OP1.2a and OP1.2b. The sensitivity of the diagnostic system as a function of wavelength, in units of W/ ( $\mathrm{m}^{2} \AA$ sr count), 


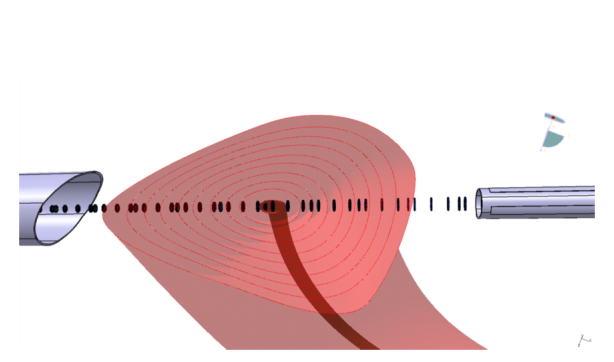

(a)

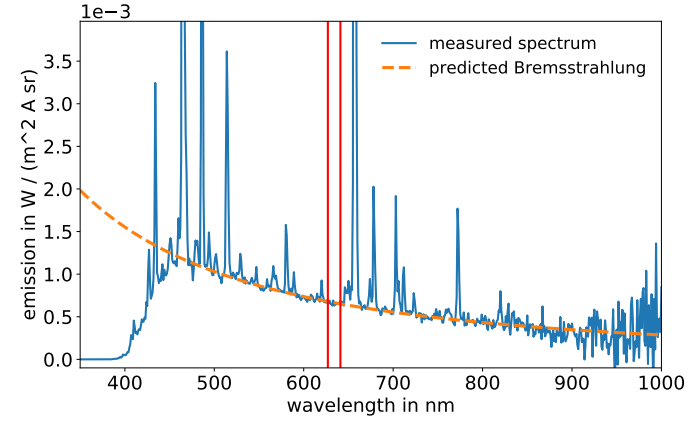

(b)

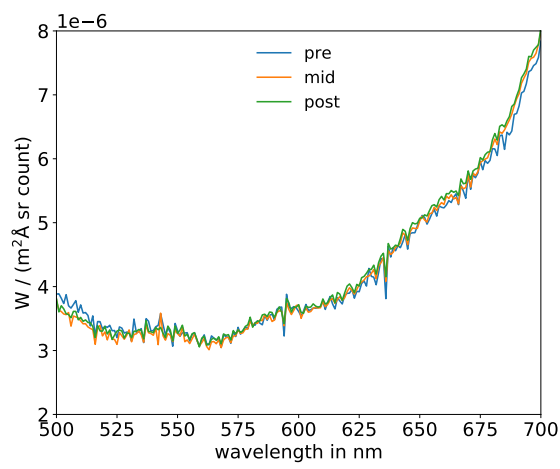

(c)

Figure 1: Figure (a) shows the single line of sight of the USB-spectrometer, the triangular W7-X plasma cross section and the magnetic axis in red. The line of sight ends in an opposite port (left hand side) thus eliminating the problem of plasma light reflections at the vessel walls. Figure (b) shows the measured spectrum of the photon flux of the plasma bremsstrahlung and line radiation (blue) as well as the predicted bremsstrahlung level with a $Z_{\text {eff }} \approx 1.5$ (dashed line). The two red vertical lines indicate the wavelength range selected and used in the analysis $\approx 627-641 \mathrm{~nm}$. Figure (c) shows the sensitivity spectrum of the diagnostic in the wavelength range of between $500 \mathrm{~nm}$ and $700 \mathrm{~nm}$. Three different measurements were carried out, prior (pre), during (mid) and after (post) the experimental campaign OP1.2.

is shown in figure 1c. Multiplication by this quantity converts the measured raw data to spectral power density in absolute units (see also $1 / C(\lambda)$ in equation 3.1). According to the time interval in which the data were collected, the corresponding calibration curve is applied to the data. The relative variation between the different curves is always $<10 \%$ in the wavelength range shown in the figure, indicating that the calibration remained fairly constant during the course of the campaign.

\section{Bayesian modeling and inference}

A model to calculate the bremsstrahlung emission is implemented in the Minerva framework [5]. The Minerva framework allows to carry out Bayesian modeling and inference in complex systems. The expected measured signal $S(\lambda)$ can be calculated from the bremsstrahlung emission at a given wavelength $V(\lambda)$ collected along the line of sight, according to equation 3.1: 

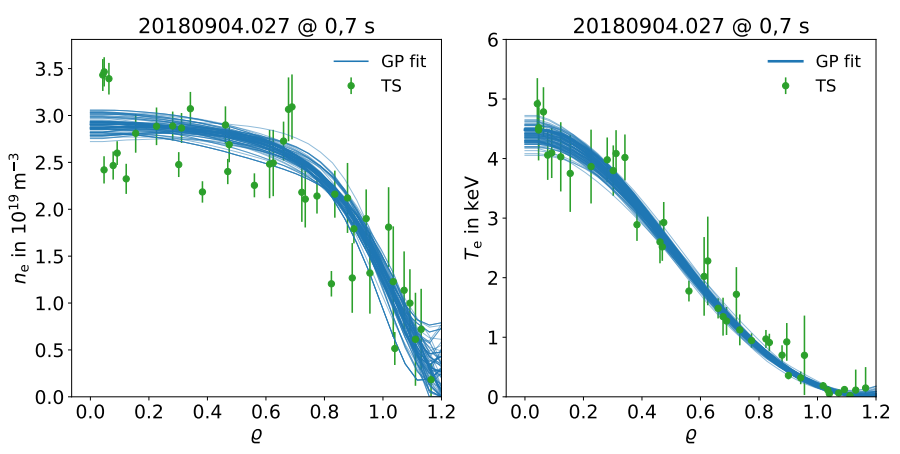

Figure 2: The electron temperature and density profiles measured by the Thomson scattering diagnostic (TS) are fitted with a Gaussian process (GP) model within the Minerva framework. The blue lines are samples from the posterior distribution found with Bayesian inference. The dots represent the measured data points together with their respective error bars.

$$
S(\lambda)=C(\lambda) V(\lambda)=C(\lambda) \int g_{\mathrm{ff}}\left(Z_{\mathrm{eff}}, T_{\mathrm{e}}, \lambda\right) \frac{n_{\mathrm{e}}^{2} Z_{\mathrm{eff}}}{\sqrt{k_{b} T_{\mathrm{e}}}} \exp \left(\frac{h c}{\lambda k_{b} T_{\mathrm{e}}}\right) \frac{1}{\lambda^{2}} d l
$$

where the integration is done along the line of sight path, $g_{\mathrm{ff}}\left(Z_{\mathrm{eff}}, T_{\mathrm{e}}, \lambda\right)$ is the free-free Gaunt factor modeled in Minerva according to [6], $C(\lambda)$ is an absolute calibration factor (figure 1c), and the remaining symbols are used in the conventional way referring to the respective physics constants in SI units. The single line of sight diagnostic does not allow to resolve the spatial profile of $Z_{\text {eff }}$, therefore, when $Z_{\text {eff }}$ is used in the calculation of the emission along the line-of-sight, it is assumed to be constant.

According to equation 3.1, $n_{\mathrm{e}}$ and $T_{\mathrm{e}}$ are quantities required to calculate the expected emission. They are provided by a spatially resolved Thomson scattering [7] measurement and a line-integrated $n_{\mathrm{e}}$ measurement by the dispersion interferometer diagnostic [8], which constrains the $n_{\mathrm{e}}$ absolute values. Both profiles are first inferred within the Minerva framework with a Gaussian processes (GP) Bayesian model [9], where the covariance of the normal prior distributions of the profiles is modeled with a covariance function, parametrised in terms of the profile length scale. The posterior solution found is "smooth" and it is affected by the number of observed data points and their respective uncertainties, which in this case do not include systematic errors. An example case of such procedure is shown in figure 2, where the samples from the posterior distribution are shown in blue and the measured data points are labeled as TS. The coordinate on the $\mathrm{x}$-axis is the effective radius $\rho=\sqrt{\psi / \psi_{\mathrm{LCFS}}}$, where $\psi$ is the magnetic flux and $\psi_{\mathrm{LCFS}}$ is the magnetic flux at the last closed flux surface.

Since the Bayesian analysis is meant to be carried automatically after every plasma discharge, a fallback solution is provided for those cases in which Thomson scattering measurements are not available. The $n_{\mathrm{e}}$ profile is assumed to be parabolic, and absolute values are scaled accordingly using interferometer measurements, as previously mentioned; the $T_{\mathrm{e}}$ profile, on the other hand, is obtained from measurements by the electron cyclotron emission (ECE) diagnostic using the cold resonance approximation $[10,11]$. 


\section{Results}

$Z_{\text {eff }}$ can be inferred by comparing predicted and measured bremsstrahlung emission signals. Two example cases from experiments 20180920.042 and 20181016.023 are depicted in figure $3 \mathrm{a}$ and $3 \mathrm{~b}$, showing discharges in which the plasma was seeded by $\mathrm{Ne}$ and $\mathrm{N}_{2}$, respectively [12]. Ne was injected at 5, 7, 9 and $11 \mathrm{~s}$ for $200 \mathrm{~ms}$, whereas $\mathrm{N}_{2}$ was injected at $2 \mathrm{~s}$ for $50 \mathrm{~ms}$ and continuously at $3-7 \mathrm{~s}$; a second valve was open at $5-7 \mathrm{~s}$, increasing the gas flow rate by a factor of $\approx 2$. Corresponding to the injection times, we observe an increase in the $Z_{\mathrm{eff}}$ values and in the total plasma radiation measured with a bolometer [13]. The $Z_{\text {eff }}$ error bars are obtained taking into account signal statistics, the absolute calibration and the uncertainties in the $n_{\mathrm{e}}$ and $T_{\mathrm{e}}$ profiles. In the figures, the time evolution of other relevant parameter is also shown: the power from the electron cyclotron resonance heating $(\mathrm{ECRH})$, the line integrated density $n_{e}$ measured with the interferometer, and the value of the electron temperature in the core $T_{e}$ as measured with the ECE diagnostic.

In figure $3 \mathrm{c}$ we show the case of a very low density discharge demonstrating the lower sensitivity limit of the diagnostic at $100 \mathrm{~ms}$ integration time. A line-of-sight averaged density $n_{\mathrm{e}}$ of 0.75 $\times 10^{19} \mathrm{~m}^{-3}$ as measured with the interferometer at $\approx 0.5 \mathrm{~s}$ allowed to measure a $Z_{\text {eff }} \approx 3.2$ with a statistical error of $\approx 50 \%$. In the second plot from the top, the measured signal for each pixel in the considered wavelength range is show at every time point; the large noise level is clearly visible.

The $Z_{\text {eff }}$ values inferred with the USB-spectrometer were also compared to those found with the charge exchange recombination spectroscopy (CXRS) system [14, 15] for two discharges 20180927.042 and 046 in which He was injected during the experiments and the neutral beam injection (NBI) system was active. According to a preliminary analysis, the CXRS system allowed to measure a $\mathrm{H} / \mathrm{He}$ ratio of $0.3 / 0.7$ and $0.85 / 0.15$ in the first and second discharge respectively, and a $2 \%$ concentration of $\mathrm{C}^{6+}$ in the core in both experiments. From these values, the lower limit of $Z_{\text {eff }}$ was then estimated as $\approx 2.1$ and 1.7 , compared to $1.9 \pm 0.4$ and $1.5 \pm 0.3$ as found with the USB-spectrometer.

\section{Conclusions and future works}

A compact, single line-of-sight USB-spectrometer allows to measure bremsstrahlung emission and infer $Z_{\text {eff }}$ at the Wendelstein 7-X stellarator. The diagnostic was operating during the OP1.2 routinely providing the line-of-sight averaged $Z_{\text {eff }}$. A Bayesian model was implemented in the Minerva framework, allowing to infer $Z_{\text {eff }}$ and to provide Gaussian process fits of $n_{\mathrm{e}}$ and $T_{\mathrm{e}}$ profiles combining measurements of the Thomson scattering and dispersion interferometer measurements.

In the context of future works, we want to mention that more systems dedicated for the $Z_{\text {eff }}$ measurement are also available and collected data during the experiments, but they are not yet modeled and inference was not carried out on such measurements. Specifically, two additional detectors share the same line of sight of the USB-spectrometer. One collects light emitted in the near infrared range of 750-950 nm, with spectral resolution of $\approx 1 \mathrm{~nm}$ and typical time resolution of $50 \mathrm{~ms}$. A second one collects visible light at $523 \mathrm{~nm}$ and $630 \mathrm{~nm}$ using interference filters with a bandwith of $2 \mathrm{~nm}$, and time resolution of $100 \mathrm{kHz}$. A third system is equipped with 27 lines of sight and operate in the range $750-950 \mathrm{~nm}$, and can therefore provide information to infer spatially resolved $Z_{\text {eff. }}$ In future works we aim at modeling all these systems and adding other diagnostics 

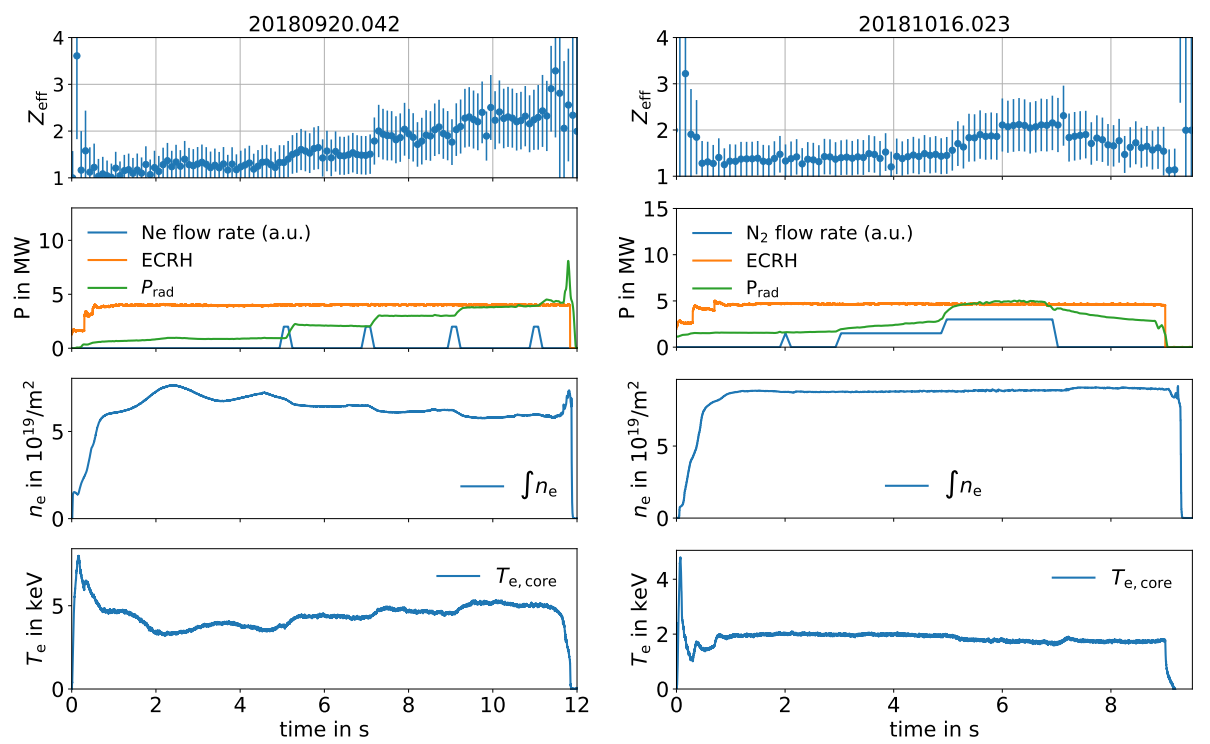

(a) A case of Ne injection.

(b) A case of $\mathrm{N}_{2}$ injection.
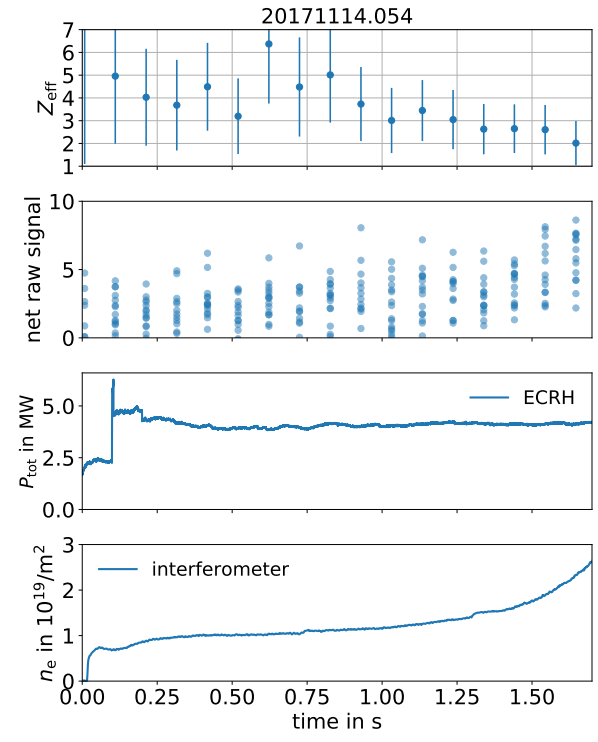

(c) A low density discharge.

Figure 3: The time evolution of $Z_{\text {eff }}$ with respective error bars and other relevant parameters for three example cases. Figures (a) and (b) show the case of two seeding experiments, with $\mathrm{Ne}$ and $\mathrm{N}_{2}$ respectively. A discrete increase in $Z_{\mathrm{eff}}$ and total radiated power $P_{\mathrm{rad}}$ is observed after each injection of seeding gas. The low density discharge in figure (c) allowed to assess the sensitivity of the diagnostic: a line-of-sight averaged density of $0.75 \times 10^{19} \mathrm{~m}^{-3}$ at $0.5 \mathrm{~s}$ allowed to measure a $Z_{\text {eff }} \approx 3.2$ with a statistical error of $\approx 50 \%$ 
containing information on $Z_{\text {eff }}$ (as CXRS, $X$-ray spectrometers) within the Minerva framework, so to exploit all available information for the inference of $Z_{\mathrm{eff}}$ profiles.

\section{Acknowledgments}

This work has been carried out within the framework of the EUROfusion Consortium and has received funding from the Euratom research and training programme 2014-2018 and 2019-2020 under grant agreement No 633053. The views and opinions expressed herein do not necessarily reflect those of the European Commission. This work was supported in part by the U.S. Department of Energy (DoE) under grant DE-SC0014210 and funding by the Department of Engineering Physics and of the College of Nuclear Engineering at the University of Wisconsin - Madison, USA.

\section{References}

[1] G. Verdoolaege, M. G. Von Hellermann, R. Jaspers, M. M. Ichir and G. Van Oost, Integrated bayesian estimation of zeff in the textor tokamak from bremsstrahlung and cx impurity density measurements, AIP Conference Proceedings 872 (2006) 541-548, [https://aip.scitation.org/doi/pdf/10.1063/1.2423317].

[2] M. Krychowiak, R. König, T. Klinger and R. Fischer, Bayesian analysis of the effective charge from spectroscopic bremsstrahlung measurement in fusion plasmas, Journal of Applied Physics 96 (2004) 4784-4792, [https://doi.org/10.1063/1.1787135].

[3] H. Y. Zhou, S. Morita, M. Goto and M. B. Chowdhuri, Zeff profile measurement system with an optimized czernyturner visible spectrometer in large helical device, Review of Scientific Instruments 79 (2008) 10F536, [https://doi.org/10.1063/1.2953489].

[4] H. Meister, R. Dux, L. D. Horton, B. Kurzan, H. Zohm and P. J. McCarthy, An integrated system to measure the effective charge of fusion plasmas in the asdex upgrade tokamak, Review of Scientific Instruments 74 (2003) 4625-4633, [https: //doi .org/10.1063/1.1605265].

[5] J. Svensson and A. Werner, Large Scale Bayesian Data Analysis for Nuclear Fusion Experiments, IEEE International Symposium on Intelligent Signal Processing (2007) 1-6.

[6] R. S. Sutherland, Accurate free-free Gaunt factors for astrophysical plasmas, Monthly Notices of the Royal Astronomical Society 300 (1998) 321-330.

[7] S. Bozhenkov, M. Beurskens, A. D. Molin, G. Fuchert, E. Pasch, M. Stoneking et al., The thomson scattering diagnostic at wendelstein 7-x and its performance in the first operation phase, Journal of Instrumentation 12 (oct, 2017) P10004-P10004.

[8] J. Knauer, P. Kornejew, H. T. Mora, M. Hirsch, A. Werner and R. Wolf, A new dispersion interferometer for the stellarator wendelstein 7-x, in 43 rd European Physical Society Conference on Plasma Physics, Leuven, Belgium P, vol. 4, 2016.

[9] C. E. Rasmussen and C. K. I. Williams, Gaussian Processes for Machine Learning. The MIT Press, 2006.

[10] M. Hirsch, U. Höfel et al., Ece diagnostic for the initial operation of wendelstein 7-x, EPJ Web Conf. 203 (2019) 03007.

[11] U. Hoefel, M. Hirsch, S. Kwak, A. Pavone, J. Svensson, T. Stange et al., Bayesian modeling of microwave radiometer calibration on the example of the wendelstein 7-x electron cyclotron emission 
diagnostic, Review of Scientific Instruments 90 (2019) 043502,

[https://doi.org/10.1063/1.5082542].

[12] F. Effenberg et al., First demonstration of radiative power exhaust with impurity seeding in the island divertor at wendelstein 7-x, Nuclear Fusion (2019) forthcoming.

[13] D. Zhang, R. Burhenn, R. Koenig, L. Giannone, P. A. Grodzki, B. Klein et al., Design criteria of the bolometer diagnostic for steady-state operation of the w7-x stellarator, Review of Scientific Instruments 81 (2010) 10E134, [https://doi.org/10.1063/1.3483194].

[14] O. Ford and other, Charge exchange recombination spectroscopy at wendelstein 7-x, Review of Scientific Instruments (2019) forthcoming.

[15] L. Vanó and other, Studies on carbon content and transport with charge exchange spectroscopy on $w 7-x$, EPS Proceedings (2019) forthcoming. 